\title{
Viewing direction and pictorial representation
}

\author{
HANS WALLACH and VIRGINIA SLAUGHTER \\ Swarthmore College, Swarthmore, Pennsylvania
}

\begin{abstract}
During prolonged monocular observation from afar, an upright wire cube (Necker cube) or a drawing of such a cube inverts continuously so that the lower of the two frontal faces appears to be either in front (version A) or in back (version B). Version A is perceived as if it were viewed obliquely from above and version $B$ as if seen from below. In our experiments, timing the durations of the versions showed that version $A$ lasted longer than version $B$. When a wire cube was shifted upward so that it was actually viewed slightly from below, version B lasted longer than version $A$. The actual viewing direction was apparently taken into account by the subjects, which was not the case when the cube was replaced by a drawing showing the projection of the cube. In that case, version A always lasted longer, regardless of the actual viewing direction. This finding conforms with picture viewing in general, where a three-dimensional object's orientation does not change when the observer's viewing direction changes.
\end{abstract}

Pictorial representation has raised many problems, for both artists and psychologists, which have been exhaustively explored in Hagen (1980). The experiment we report here addressed a specific question: Does picture perception follow rules that are different from those of space perception? To answer this question, we need to examine the conditions of stimulation that are present in picture perception but not in ordinary space perception.

One important instance of such conditions occurs when a picture that represents a tridimensional scene is looked at from an oblique direction. When the objects represented in the picture are looked at in real space and when they are seen from a different angle, the arrangement of objects is different; the orientation of the scene relative to the line of sight has changed. But when one makes the same change in viewing direction while facing the picture of the same scene, the arrangement does not change. The retinal projection of the picture is merely given with a form distortion.

Wallach and Marshall (1986) showed that a compensation for this distortion takes place that resembles shape constancy. This compensation may be a process that is specific to picture perception, but, as Wallach and Marshall pointed out, there is an alternative explanation. Ordinary shape constancy, which corrects for the foreshortened projections of slanted surfaces, may result in a representation of the picture that is structured as if the picture were seen in perpendicular view. This perceptual stage would subsequently be subjected to the configurational process that constructs the perceived tridimensional scene from the corrected representation of the picture's retinal projection. Postulating such a two-stage process

This work was supported by Grant BSN-8318772 from the National Science Foundation to Swarthmore College, Hans Wallach, principal investigator. The author's mailing address is Department of Psychology, Swarthmore College, Swarthmore, PA 19081. makes it possible to avoid invoking a compensation process that is specific to picture perception.

The experiment reported here is another attempt to show that perception of tridimensional objects in pictures is different from perception of tridimensional objects in ordinary space. As before, a picture with tridimensional content was viewed from an oblique direction-a condition that would change the orientation of the contents if they appeared in real space but would have no such result when the contents were shown as a picture. This time we found that a change of vantage point has a particular effect in actual space perception but no such effect when the objects are viewed in a picture.

Our object was a wire cube, which, when observed monocularly, would readily invert. Figure 1, which depicts the projection of the cube as presented to our subjects, shows two square faces of the cube in frontal orientation. Two arrangements in depth can be perceived, with the lower face being either in front (version A) or in back (version B). Version A implies an oblique view from above, whereas version $B$, with the lower face in back, implies an oblique view from below. During prolonged observation, subjects saw these two versions alternate, with version $A$ usually being perceived for longer periods than version $B$. The advantage of version $A$ can be attributed to a memory effect; orthogonal objects that resemble the cube are more often seen obliquely from above than from below. It seemed possible, however, that, in addition to the preference for version $\mathrm{A}$ caused by the memory effect, the actual viewing direction might also affect perception, and that was found to be the case; somewhat elevating the cube so that it was seen obliquely from below favored version $B$, in concordance with that viewing direction. We also hoped to find that the effect of viewing direction would be absent when the wire cube was replaced by a picture of the cube, since a change in viewing direction might not have the same effect in picture perception as it has in space perception. As we have seen, 
when the viewing direction is changed from perpendicular to oblique, a tridimensional scene is rearranged, whereas a picture of that scene is not; instead, the whole picture is foreshortened. To show that elevating the cube does not favor version $B$ when the real cube is replaced by a picture of the cube, we obtained the difference in the durations of versions $A$ and $B$ for a cube in both elevated and lowered positions and then compared that difference with the corresponding difference for pictures of the cube in the same positions.

\section{METHOD}

\section{Subjects}

Forty-six paid undergraduates from Swarthmore College served as subjects.

\section{Equipment}

Two identical $5-\mathrm{cm}$ wire cubes were mounted in front of a translucent screen, one $23 \mathrm{~cm}$ above the subject's eye level and the other $25 \mathrm{~cm}$ below. Each subject sat with his or her head in a headrest that was adjusted to maintain the same eye levels for different subjects. The distance between the subject's eyes and the center of the cube was $210 \mathrm{~cm}$, far enough for distortion of polar perspective to be not noticeable. One cube's angle of regard was $6.3^{\circ}$ above and the other was $6.5^{\circ}$ below the horizontal plane. The cubes were oriented so that their projections at the subject's eye were exactly the same. The cubes were viewed with one eye only and were presented one at a time. A spotlight illuminated a circular area of the translucent screen behind the exposed cube, and a small piece of cardboard was inserted in front of the other cube to hide it.

A drawing of the cube was prepared that showed the same projection as the real cubes (Figure 1). When the drawing was presented, the cubes were removed and the screen was replaced by a piece of cardboard. The drawing was attached in either the upper or lower position and was illuminated from the front. To make sure that the subjects perceived it as a picture, they observed the drawing binocularly. Also, a frame consisting of a 2 -mum-wide line was drawn around the cube. This frame was quite large $-22 \times 21 \mathrm{~cm}-$ so that its retinal projection was outside the macula.

To enable the subjects to report which version of the cube they saw, a small bump was fastened to one of the vertical wires of the cube. It consisted of a narrow piece of plastic tape that was wound around the wire. We preferred to have the subjects report whether the bump was at the front or the back of the cube rather than whether the cube's lower face was in front or in back, which might have made them more aware of the different viewing directions that the two versions implied. For half of the subjects, the bump was attached to the front face of the cube; the remaining subjects saw the cube with the rear face marked. We made a corresponding arrangement for the drawing: Two drawings were prepared, one with

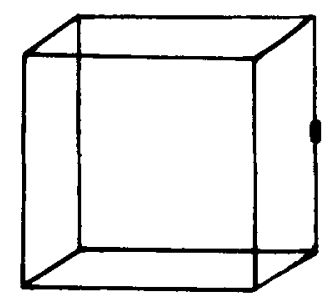

Figure 1. Projection of the wire cube in both of the display positions and in one of the drawings of the cube.
Table 1

Means of the Total Times (in sec) for Version A (Perceived as if Seen from Above) and Version B (Perceived as if Seen from Below) during an Observation Period of $120 \mathrm{Sec}$

\begin{tabular}{cccccc} 
& \multicolumn{2}{c}{ Real Cubes } & & \multicolumn{2}{c}{ Drawing } \\
\cline { 3 - 5 } Real Position & Low & High & & Low & High \\
\hline Version A & 65.8 & 58.1 & 70.4 & 80.0 \\
Version B & 54.1 & 61.8 & 49.5 & 39.9 \\
\hline
\end{tabular}

the bump on the lower face and the other with the bump on the upper face.

\section{Procedure}

A group of 23 subjects observed the inversions of the wire cubes. They were told that they would be looking at a three-dimensional wire cube with a small bump affixed to one of the side bars of the cube. The bump would appear to be either at the front of the cube or at the back of the cube, and they were to watch the cube for $2 \mathrm{~min}$, all the while reporting whenever the bump appeared to move from the front to the back of the cube, or vice versa. The subjects were to respond by saying "front" or "back" whenever they saw the bump move, an indication of the cube's inversion. The experimenter timed the reported changes by pressing a key on her computer, which tabulated the number and length of the intervals for each version of bump location. Each subject went through this procedure twice, once with the upper and again with the lower cube.

Another group of 23 subjects observed the inversions in the drawings, once with the drawing in the upper position and again with the drawing in the lower position. The procedure was the same as the one that dealt with the real cubes, except that the subjects observed the drawing binocularly and the instructions referred to a drawing of a cube. Half of the subjects who observed the real cubes reported the changes of the elevated cube first and then those of the cube in the lower location. For the other subjects, this order was reversed. Obtaining the time course of the changes in the drawings in the two positions was designed in the same way.

\section{RESULTS}

Table 1 shows the mean totals of time, in seconds, that the subjects reported seeing versions A and B during the 2 -min observation period. The results for subjects who reported the inversions of the real cube-really the changes in the apparent location of the bump-show an influence of the viewing direction: Version $\mathrm{A}$, which was arranged as if it were seen from above, lasted longer when the cube being observed was in the lower position, and version $B$ lasted longer when the cube being observed was in the upper position, where, in fact, it was seen obliquely from below. This difference was statistically significant $[t(22)=2.50, p=.02]$. As was hoped, this effect of actual viewing direction was absent in the group of subjects who observed the drawing of the cube. The total duration of each version was the same for both positions of the drawing. On the other hand, the tendency to favor perception of version A was strong. This agrees with casual findings made by the senior author while teaching. When he demonstrated the Necker cube, the great majority of observers saw version $A$ of the cube first, as did our subjects. A statistical test of the difference of the results for the real cubes and for the cube drawings showed that it was significant. For the subjects who 
observed the real cubes, we obtained the difference between the total times during which they saw version A when the cube was in the high position and when it was in the low position. For the subjects who observed the drawing, we computed the corresponding difference scores. The two means of these difference scores differed $[t(44)=2.81, p<.01]$.

\section{DISCUSSION}

We found that when the subjects observed a wire cube, the alternation between the perceived versions of the cube when rendered in parallel perspective was influenced by the viewing direction. Viewing direction, however, had no such effect when subjects were shown a picture of a cube that provided the same retinal projection as the real cube.

This finding agrees with the conditions that prevail in space perception and in picture perception. When observers change vantage point while viewing a real tridimensional scene, their retinal image of an object in that scene represents it as having undergone a small rotation. However, when a picture of that scene is viewed with the same change in viewing direction, there is no change in the orientation of the object; the projection of the object is merely foreshortened. ${ }^{i}$ This difference in the effect of changed viewing direction seems to go hand in hand with a different way in which picture perception operates. Since, with a picture, changes in viewing direction do not alter an object's orientation to the line of sight, changes in viewing direction need not be taken into account. ${ }^{2}$ The failure of viewing direction to influence the durations of the cube versions shows that it is disregarded.

Our experiment also shows that, when a picture is seen, this effect is not a consequence of an absence of change in orientation when the viewing direction is changed. When the real cubes were observed, there was also no change in orientation; the cubes were arranged so that their retinal images were the same for both viewing directions. Nevertheless, viewing direction had an effect on the duration of the versions of the real cubes.

It has been pointed out to us that our interpretation of these results seems to conflict with a common observation: that as one moves past a picture in which a tridimensional object or a scene with strong depth is realistically rendered, such objects or scenes seem to rotate. Wallach, Stanton, and Becker (1974) have explained these observations as being the result of a process-applied to picture contents-that compensates for the relative rotation of objects or scenes when one moves in relation to them. Wallach et al. demonstrated the existence of such a compensation process by showing that a stationary object that undergoes the normal relative rotation caused by the observer's movement will appear stationary, but that an object whose relative rotation is larger or smaller than normal-on the average by more than $40 \%$ of the rela- tive rotation-will appear to rotate. In Wallach et al.'s experiments, such abnormal relative rotations were produced by having the object actually rotate under the control of the observer's change in position. The picture content, by not rotating at all, is equivalent to an abnormal relative rotation of a magnitude of $100 \%$ and will cause perceived rotation.

Thus, the compensation process that causes the apparent rotation of picture contents seems to take position change into account, whereas in the present experiment, differences in viewing direction were not taken into account when the cubes were represented in pictures. How can the two cases be reconciled? The apparent rotation of the picture content results from one of a number of processes that compensate for the visual stimulation caused by the observer's movements (Wallach, 1987). In such compensation processes, proprioceptive representation of the observer's movements and the visual stimulation that the movements cause are being matched up, which is also the case with the relative rotation of objects caused by the observer's translatory movements, as Wallach et al. (1974) have shown. That the observer's movement also caused changes in viewing direction may play no role in the compensation process that causes apparent rotation of the picture content. Changes in viewing direction are not taken into account in picture perception, which may therefore not prevent the apparent rotation of picture contents that results from the compensation process. Sometimes different perceptual functions, because they operate independently of each other, have paradoxical results. ${ }^{3}$ The matching-up process involved in compensation for movement-produced stimulation is quite different from the process involved in learning that changing viewing direction does not have the effect in viewing pictures that it has in viewing real tridimensional scenes.

There may be another perceptual function in which picture and real space perceptions differ. If another such difference can be demonstrated, it may make sense to speak of picture perception as a separate mode of perception. At any rate, the present experiment suggests that two ways of perceiving a tridimensional object that result from the same retinal projection exist side by side. They depend on whether the distal stimulus is accepted as being tridimensional or whether cues, such as the absence of retinal disparity in binocular viewing, operate that represent the distal stimulus as a picture.

\section{REFERENCES}

DEESE, J. W. (1966). Moon illusion and size-distance invariance: An explanation based on an experimental artifact. Perceptual \& Motor Skills, 23, 629-630.

HAGEN, M. A. (1980). The perception of pictures. New York: Academic Press.

Wallach, H. (1987). Perceiving a stable environment when one moves. Annual Review of Psychology, 38, 1-27.

Wallach, H., \& MarShall, F. J. (1986). Shape and constancy in pictorial representation. Perception \& Psychophysics, 39, 233-235. 
Wallach, H. Stanton, L., \& Becker, D. (1974). The compensation for movement-produced changes in object orientation. Perception \& Psychophysics, 15, 339-343.

\section{NOTES}

1. Foreshortening becomes noticeable only when the viewing direction is shifted by a sizeable angle. In our experiment, where this shift amounted to $6.5^{\circ}$, foreshortening amounted only to $0.64 \%$ and could not have been noticed.

2. Only the orientation of the projection of the object changes, which, if it is large enough, causes a foreshortening of the projection. The compensation that deals with it may or may not be a characteristic of picture perception (Wallach \& Marshall, 1986)

3. A well-known example of paradoxical results caused by mutually independent processes is the appearance of the moon when it is at the horizon. It appears large and near. It seems large because it is given with cues for being at a large distance (Deese, 1966), the result of the operation of the law of size perception. And it appears to be near because it looks large, a result of the dependence of apparent distance on the size of familiar objects. An analogous observation can be made when spectacles are worn that cause oculomotor adjustments to be made for distances larger than the actual distances of the seen objects. A familiar object looks too large because it is given with cues for an abnormally large distance, and it paradoxically looks near because its perceived size is large.
(Manuscript received December 2, 1986; revision accepted for publication July 31,1987 .) 